\title{
Stratifying patients for polypharmacy interventions: the case for a new biomarker?
}

\author{
Christopher Threapleton ${ }^{1}$, Tess Harris ${ }^{2}$, and Emma Baker ${ }^{1}$ \\ ${ }^{1}$ St George's University Hospitals NHS Foundation Trust \\ ${ }^{2}$ St George's University of London
}

September 25, 2021

\section{Hosted file}

Commentary on Bengaard paper July 2021 FINAL DRAFT.docx available at https://authorea.com/ users/436475/articles/538765-stratifying-patients-for-polypharmacy-interventions-thecase-for-a-new-biomarker 